\title{
Transparenz, Teilhabe und introjektive Identifizierung
}

\author{
Karl-Albrecht Dreyer
}

Online publiziert: 4. Mai 2020

(C) Springer Medizin Verlag GmbH, ein Teil von Springer Nature 2020

Zusammenfassung In diesem Beitrag wird eine reflektierte Offenheit des Analytikers als zentrale Haltung zur Förderung des psychoanalytisch-psychotherapeutischen Prozesses beschrieben. Austausch und Reflexion werden wesentlich erleichtert. Transparent vorgehende Psychoanalytiker und Psychotherapeuten fördern damit die Teilhabe ihrer Analysanden/Patienten an der analytischen Arbeit. Dadurch lassen sich in der Kindheit entstandene pathogene introjektive Identifizierungen erkennen und auflösen. Die Arbeit stellt nach einer Klärung der Begriffe „Transparenz“ und „Teilhabe“ und ihres theoretischen Hintergrunds in einem Fallbeispiel die typische, mit introjektiver Identifizierung verbundene Interaktion in ihrem Ablauf und der jeweiligen inneren Dramaturgie der Beteiligten dar. Der Vorgang der introjektiven Identifizierung überbrückt in der analytischen Situation die Kluft zwischen unbewussten Themen im Analysanden und im Analytiker: Zunächst unbewusste Bilder und Vorstellungen können über einen fruchtbaren Klärungsprozess der Analyse zugänglich gemacht, ihr Entstehungsort und ihre Entstehungsdynamik geklärt werden. Transparenz und Teilhabe sind wertvolle technische Mittel, um den unbewussten Teil von introjektiven Identifizierungen in all seinen Verästelungen zu entdecken und zu verstehen.

Dr. med. K.-A. Dreyer $(\bowtie)$

St.-Leonhard-Str. 7, 89077 Ulm, Deutschland

E-Mail: dreyer.ka@t-online.de 


\section{Transparency, participation and introjective identification}

Abstract In this article a transparency of the analyst is seen as a key aspect with respect to the development of the psychoanalytic psychotherapeutic process. Psychoanalysts and psychotherapists with a transparent approach enable their analysands/ patients to participate in the course of the analytic work. Transparency and participation jointly facilitate the shared reflection on internal and interactive processes. These processes may take the form of introjective identification. Transparency and participation create an atmosphere in which the dyad has a better grasp of unconscious aspects in the introjective identification. After clarification of the terms transparency and participation and the theoretical background, a case report is presented showcasing crucial interactions in which the mechanism of introjective identification and the internal dramaturgy of both participants is demonstrated. The case report is connected to the theoretical part in the concluding remarks. In the analytic situation the process of introjective identification bridges the gap between the analysand's and the analyst's unconscious themes: as a result the psychoanalyst can discover unconscious affects reaching the inner world originally stemming from the unconscious of the analysand/patient. A fertile interplay emerges within the analyst, which can be communicated to the analysand and is then present in the dyadic analytic experience. Transparency and participation are valuable technical means to discover and understand the unconscious part of introjective identification in all its ramifications.

\section{Einleitung}

Eine Haltung als Psychoanalytiker und Psychotherapeut, die auf Transparenz und Teilhabe achtet, verändert die Perspektive auf den psychoanalytischen Prozess. Dabei ist zu erfahren, welch wertvolle Begleiter Transparenz und Teilhabe sind: Unseren Patienten und Analysanden wird es leichter, uns an ihren Gedanken und Gefühlen teilhaben zu lassen - eine für den Therapieerfolg unabdingbare Voraussetzung. Es entsteht ein Dialog, der von der Dyade gemeinsam hervorgebracht und getragen wird. Im therapeutischen Prozess kann über spezifische Übertragungsmomente und deren Einfluss auf die Beziehung kommuniziert werden. In dieser Arbeit möchte ich zeigen, welcher Gewinn aus einer transparenten Haltung in reflektierter Offenheit und wechselseitiger Teilhabe entsteht und welche weiterführenden Möglichkeiten sich daraus ergeben. Das führe ich am Beispiel der introjektiven Identifizierung aus. Wenn ich in der männlichen Form schreibe, meine ich beide Geschlechter.

Transparenz entsteht, wenn der Patient die Grundregel der freien Assoziation als Aufforderung zu Offenheit ernst nimmt und der Therapeut in reflektierter Offenheit nach außen und nach innen, in gleichschwebender Aufmerksamkeit und gebotener Abstinenz die unbewussten Prozesse zu verstehen und zu interpretieren sucht. Diesen letzten Teil fasse ich nochmal kürzer: Transparenz entsteht aus der reflektierten Offenheit des Therapeuten. Nach meinem Verständnis verlangt dies bisweilen auch, dass der Therapeut sein Empfinden mitteilt. Sein Schweigen kann, auch wenn es abstinent intendiert ist, in manchen Momenten manipulativ wirken. Luiz (2006) gibt ein eindrucksvolles Beispiel einer notwendig transparenten Behandlung 
wieder. Transparente Momente entstehen in Behandlungen besonders dann, wenn überraschende Begegnungen, die beispielsweise in ,now moments“ (Stern 2010) entstehen, analysiert werden.

Teilhabe wird wechselseitig verstanden: Der Analysand lässt seinen Analytiker an seinen Assoziationen teilhaben. Der Analytiker wiederum lässt seinen Analysanden an seinen Überlegungen und Empfindungen insoweit teilhaben, wie diese für den analytischen Prozess fruchtbar, sinnvoll und zur Klärung notwendig sind. Teilhabe ist also ein Prozess in zwei Richtungen: vom Patienten in Richtung auf den Therapeuten und vom Therapeuten in Richtung auf seinen Patienten. Beide nehmen aktiv teil und lassen passiv an sich teilhaben. Diesen Vorgang beschreiben Stolorow und Atwood (1992) aus relationaler Sicht, Ferro (2005) unter dem Gesichtspunkt des Feldes, das Bohleber (2014, S. 66) in ein kontinuierliches, gemeinsames Träumen, das Wachtraumdenken bei Bion (1962), auflöst und das von Ogden (2006, S. 61) revolutionär neu in einer passageren Auflösung der Subjektgrenzen konzipiert wird.

\section{Intersubjektivität, aber welche? Abstinenz, aber wie?}

Das große Feld der intersubjektiven und relationalen Psychoanalyse präsentiert sich vielgestaltig. Altmeyer und Thomä (2006) nennen ihr Buch Die vernetzte Seele im Untertitel Die intersubjektive Wende in der Psychoanalyse. Die beiden Autoren gehen davon aus, dass es die Ergebnisse der Säuglingsforscher sind, die der Intersubjektivität letztlich zum Durchbruch verholfen haben. Aus einer anderen Perspektive sieht Bohleber (2014) in der kritischen Gesellschaftstheorie der 1960erJahre einen Ausgangspunkt dafür, dass sich die intersubjektive Sicht durchgesetzt hat: Die philosophischen Ansätze von Buber und Binswanger hatten großen Einfluss auf die Tiefenpsychologie in Deutschland, die zum Teil noch geprägt war vom alten Göring-Institut. Wenn auch die damaligen Ansätze bereits die fundamentale Bedeutung der Intersubjektivität betonten, gelang die Übersetzung in reale klinische Beziehungskonstellationen nicht. Zu einer Neuaufnahme der Themen um Intersubjektivität kam es ausgehend von der kritischen Gesellschaftstheorie ab den 1960erJahren: Lorenzer hebt 1970, S. 141, hervor, dass ,,sich das hier beschriebene Verstehen der Interaktion der Subjekte mit ihrer Mitwelt und Umwelt zu[wendet]“.

Ebenso lässt sich eine psychoanalytisch-intersubjektive Sicht auf dem theoretischen Fundament von Klein und Bion gründen (Böhme 2014). Und Mertens (2013) wiederum legt in seinem Aufsatz „Das Zwei-Personen-Unbewusste - unbewusste Wahrnehmungsprozesse in der analytischen Situation" den Schwerpunkt auf die unbewusste wechselseitige Einstimmung. Aber auch die Perspektive des psychoanalytischen Feldes mit sich in dieses Feld hinein auflösender Individualität, wie von Baranger und Baranger (2008) sowie von Ferro (2005) und Ogden (2006) beschrieben, kann als theoretische Untermauerung einer intersubjektiven Betrachtungsweise gewählt werden.

Intersubjektives psychoanalytisches Denken und Arbeiten wurden in der Vergangenheit durch vielgestaltige, auch außerhalb der Psychoanalyse gelegene Einflüsse befördert. Auch in der Gegenwart beeinflussen die gesellschaftlichen Entwicklungen unsere psychoanalytische Theorie: Der veränderte Umgang mit Daten und das 
wachsende Bewusstsein zur infomationellen Selbstbestimmung wirken auf unsere Theorie und Praxis ebenso wie der gewaltige Druck, der beispielsweise aufgrund einer Epidemie wie COVID-19 (,coronavirus disease 2019“) auf der Bevölkerung lastet. Daraus entstehende Konflikte lassen sich in einem demokratischen Gemeinwesen nur durch Transparenz und Teilhabe mildern. Die Begriffe „Transparenz“ und „Teilhabe“, die ich für die psychoanalytische Theorie und Behandlungstechnik vorschlage, tragen dazu bei, aktuell bedrohlichen Entwicklungen einen mit Hoffnung angereicherten sprachlichen Ausdruck gegenüberzustellen.

Eine besondere Rolle spielt in diesem Zusammenhang der Abwehr- oder Interaktionsmechanismus der introjektiven Identifizierung. Transparenz und Teilhabe helfen dabei, die unbewussten Anteile der introjektiven Identifizierung zu entschlüsseln. Den Zusammenhang und das Zusammenspiel dieser Mechanismen werde ich in der vorliegenden Arbeit theoretisch untersuchen und an einem klinischen Beispiel veranschaulichen.

Ebenso vielgestaltig wie Theorie und Praxis der Intersubjektivität ist heutzutage die Handhabung der Abstinenz. Dies ist nur folgerichtig, wenn wir uns die Geschichte der Begriffe, „Abstinenz“ und „Indifferenz“ (S. Freud 1919a) vergegenwärtigen, die aus der Zeit der Triebtheorie (S. Freud 1915b) und des zweiten topischen Modells ab 1920 (S. Freud 1923b) stammen. Die erste Zeit der Psychoanalyse war geprägt von Modellen, in denen Personen eher voneinander abgegrenzt aufgefasst wurden (S. Freud 1912e). Heute hingegen weisen die Modelle der intersubjektiven Affektivität auf Wege hin, wie durch Affekte Personengrenzen überschritten werden und Interaktionen in die Psyche des anderen hineinwirken können.

Im Jahre 1984 plädiert Cremerius für eine operationale Anwendung der Abstinenzregel als Funktion des analytischen Prozesses (1984). Ogden geht zwanzig Jahre später erheblich weiter (2006, S. 61) und spricht im Rahmen der projektiven Identifizierung von einer passageren Auflösung der Subjektgrenzen von Analytiker und Analysand. Insoweit sich die Wahrnehmung der Subjektgrenzen auflöst, ist eine Abstinenz, die diese Grenze eigentlich voraussetzt, nicht mehr eindeutig zu definieren. Zusammenfassend ergibt sich daraus nach Ermann (2014, S. 121) „,eine Neukonzeption des klassischen Abstinenzprinzips .... Aus intersubjektiver Sicht ist diese Regel, die oft sehr starr gehandhabt wurde, durch ein funktionales Prinzip ersetzt worden. Danach hat der Analytiker je nach Lage der Dinge zu entscheiden, ob, wie weit und in welcher Form er auf Wünsche und Begehren des Patienten eingeht, um anschließend zu untersuchen, wie der Patient sein Verhalten verarbeitet." Zu diesem Reflexionsprozess trägt eine Haltung von Transparenz und Teilhabe Wesentliches bei, weil durch sie die Motive der Interaktion klarer beschrieben und bearbeitet werden können.

\section{Transparenz, Teilhabe und introjektive Identifizierung}

Nach meiner Einschätzung finden im psychoanalytischen Theoriegebäude Transparenz und Teilhabe nicht die Beachtung, die ihnen aufgrund ihres Potenzials, Unbewusstes bewusst werden zu lassen, zukommt. Ich verfolge in meiner Arbeit die These, dass dies insbesondere für die Bearbeitung der introjektiven Identifizierung 
gilt. Sie kann günstigenfalls dann gelingen, wenn der Analytiker in einer Haltung arbeitet, durch die transparent wird, was gerade vor sich geht. Seit Ferenczi (1933) sind uns die Grundlagen für das Verständnis der introjektiven Identifizierung bekannt, die er in seiner berühmten Arbeit ,Sprachverwirrung zwischen den Erwachsenen und dem Kind“ gelegt hat. Es war dann Klein (1946), die den Vorgang der introjektiven Identifizierung auf den Begriff gebracht hat.

Die introjektive Identifizierung, wie wir sie heute verstehen, ist ein Abwehrund Interaktionsmechanismus, der an der Grenze zwischen Pathogenese und Salutogenese steht: Durch die initiale Introjektion ,,verschwinden“ quasi konflikthaftbedrohliche Affekte im Unbewussten der introjizierenden Person - insofern trägt der Vorgang einen pathogenetischen Charakter. Die Folgen im neurotischen Geschehen können bis zur Entwicklung eines falschen Selbst führen. Auf der anderen Seite kann in der depressiven Position eine Identifizierung des Subjektes mit den introjizierten Affekten die Tür zu einer neuen Entwicklung öffnen. Mit dem Aufscheinen eines neuen Verständnisses beginnt eine salutogenetische Wirkung der introjektiven Identifizierung.

Diese Prozesse können sowohl im individuellen Leben wie in der Psychoanalyse geschehen. Neue Erfahrungen entstehen aus einer bewussten Aneignung der zunächst unbewussten Introjektion: In der Regel nimmt im psychoanalytischen Prozess der Analytiker zuerst etwas wahr, das sich in seinem Inneren fremd, nicht zu ihm gehörend, anfühlt. Das Gefühl der Fremdheit kann längere Zeit verborgen bleiben. Erste Wahrnehmungen tauchen auf und kündigen die mögliche Bewusstwerdung an. Erst vorsichtig, dann ermutigt durch ein erstes Verstehen kommt der Weg zu einem bewussten Verständnis in Gang. In dem Maße, wie dieser Vorgang gelingt, öffnet sich ein Verständnis für umfangreiche, bisher nicht bewusstseinsfähige Zusammenhänge.

Um zusammenzufassen: Durch den Mechanismus der introjektiven Identifizierung kann von unbewusst zu unbewusst eine Brücke zwischen Analytiker und Analysand geschlagen werden. Den zentralen Unterschied der introjektiven im Vergleich zur projektiven Identifizierung fasst Schoenhals Hart (2007, S. 171) wie folgt: „Die introjektiven Identifizierungen (mit den guten Objekten) fördern die integrative Entwicklung in Richtung depressiver Position, während die projektiven Identifizierungen den Ausstieg aus dieser Entwicklung bedeuten“.

Beispiele für introjektive Identifizierungen finden wir in unseren Behandlungen häufig dann, wenn es in der Entwicklung des Kindes eine Notwendigkeit oder einen Zwang gab, empathiegeleitet, mithilfe einer introjektiven Identifizierung zu versuchen, unverständliches, verletzendes elterliches Verhalten durch Übernahme und Verinnerlichung zu entschärfen. Das Kind nimmt Unverständliches in sich auf, bildet daraus ein Introjekt und identifiziert sich damit. Die äußere Gefahr wird so durch Verinnerlichung entschärft. Das Kind verzichtet damit aber zugleich auf eigene Entwicklung und versucht stattdessen, die Eltern, die es so dringend braucht, doch noch gut genug zu machen. Ferenczi hat es in seinem Aufsatz (1933) beschrieben.

Den inneren Abbildern jener Vorgänge und dem häufig damit verbundenen Entwicklungsverzicht begegnen wir sehr viel später in unserem Behandlungszimmer. Eine Haltung empathischer Transparenz des Analytikers bietet dann die Chance, verborgene Signale aufzunehmen, introjektive Identifizierungen bewusst zu machen 
und dadurch einen Ausweg zu öffnen - diesen Weg illustriere ich nun in meiner Fallvignette.

\section{Eine Fallvignette}

Eine Vorbemerkung zum Verständnis von Vignetten: Mit Schülein (2002) gehe ich von der Psychoanalyse als einer Wissenschaft aus, die mit konnotativen Theorien arbeitet. Daher bleiben ihre Aussagen zwangsläufig offen und unscharf, an Narrative gebunden. Falldarstellungen können folgerichtig keine Beweise liefern; sie beziehen ihre Qualität aus ihrer Plausibilität und begründen ein ,Denken in Fällen“, wie dies Hampe und Strassberg beschreiben (2015, S. 281; Dreyer 2017, S. 34-39): Die Methode der psychoanalytischen Erkenntnis ,,ist eine Disziplin, die Mythen und Geschichten aus der Literatur zu Entwicklungsschemata formalisiert und sie zusammen mit wirklich erhobenen Fallgeschichten aus der psychoanalytischen Praxis zum Erkenntnishintergrund individueller Geschichten macht.“

„Als ich heute vor Ihrer Haustüre stand, wollte ich mit meinem Schlüssel aufschließen!" beginnt die Patientin heiter die Stunde; ihre Fehlleistung ist für mich ein Zeichen, dass sie bei mir eine Heimat gefunden hat. In der Stunde spricht sie über ihre aktuelle Beziehung und die Bedeutung von Sexualität. Sie fragt sich, ob ihr Partner für sie der Richtige sei. Ich stelle nach einiger Zeit überrascht fest, dass in mir Traurigkeit entsteht. Sie passt nicht zu der Stimmung und dem Tonfall der Patientin; ich kann mir mein Empfinden selbst nicht erklären. Ich bin weder bedrückt noch traurig in die Stunde gekommen. Im Zuhören bleibe ich mit dieser Diskrepanz beschäftigt und frage mich, ob ich diese im Sinne der ,negative capability“ (Bion 1970, S. 125) aushalten oder durch Aussprechen die Diskrepanz der Reflexion zugänglich machen soll. Wenn ich eine Möglichkeit wähle, verliere ich damit zugleich die andere und beeinflusse in jedem Fall den weiteren Verlauf - auch durch Schweigen.

Den Gedanken, ich könnte komplementär zu ihr die Empfindungen ihres verlassenen Freundes fühlen, spreche ich aus. Dieses Verstehensangebot gewinnt jedoch keine Bedeutung. Sehr viel später, in der Reflexion der hiesigen Veröffentlichung erkenne ich in meinem frustranen Deutungsversuch eine Parallele zum vergeblichen Versuch meiner Patientin, mit ihrem Schlüssel mein Haus aufzuschließen.

„Ich habe bemerkt, dass ich während Ihrer Schilderung traurig wurde, ohne dass ich sagen könnte weswegen“, formuliere ich dann offen. Der weitere Verlauf der Stunde nimmt eine überraschende Wendung. Wir fragen uns in der Stunde, ob ihr Beziehungsproblem mit ihrer Missbrauchserfahrung als Teenager zu tun haben könnte. Sie gebraucht im Gesprächsverlauf den Ausdruck ,dumm“, um ihre aktuelle Reaktion in der Stunde und ihr Selbstempfinden generell zu beschreiben. Ich frage nach, woraufhin sie bekräftigt: „Ich bin dumm“. Ich bitte um weitere Erläuterung. Sie findet schließlich zu einem literarischen Vergleich mithilfe des Romans 1984 von Orwell (2002). Darin würden sich die Protagonisten selbst „dumm“ machen, sagt sie. So gehe es ihr auch. Sie sei ebenso „dumm“ wie die Bürger im Roman von Orwell und betont damit den aktiven Part des Sich-selbst-Verdummens, der aktiven 
Ich-Einschränkung, von der Ferenczi 1933 schrieb. Ganz im Gegensatz zum Inhalt ihrer Überlegung finde ich ihre Erklärung ausgesprochen klug.

Nun tauchen in mir mögliche Zusammenhänge zwischen ihrem Gefühl, dumm zu sein, und meinem Gefühl von Traurigkeit auf: Ich frage mich, ob mein mir rätselhaft gebliebenes Gefühl möglicherweise ihre abgewehrten, nicht ins Bewusstsein zugelassenen Affekte enthält: Traurigkeit entsteht stellvertretend in mir, während die Patientin nach ihrer Aussage kein anderes Mittel fand, als sich selbst zu verdummen, um quälende Zusammenhänge unbewusst zu halten. Als ich diesen Zusammenhang ausspreche, beginnt für die Patientin der Weg, ihre Selbstverdummung und ihre Scham darüber zu verstehen und die lang verborgenen Emotionen wieder zu erleben. Ich denke, dass ich zunächst im Rahmen einer introjektiven Identifizierung die Trauer aus dem Unbewussten der Patientin übernahm; nur über mich konnte sie auch ihr bewusst werden.

Zum Verständnis der Vignette: Sie beginnt mit einer Fehlleistung, die wir manchmal in fortgeschrittenen Behandlungen beobachten können, wenn Patienten versuchen, mit ihrem Schlüssel die Praxis ihres Analytikers aufzuschließen. Ebenso wie die Praxis für meine Patientin bleibt mir meine Empfindung zu Beginn der Stunde verschlossen, und mein erster Deutungsversuch war ebenfalls vergeblich. Gleichzeitig wird durch die Fehlleistung der Wunsch ausgedrückt, Zugang zum Verborgenen zu finden. In der Stunde entfaltet sich ein unbewusster „Plan“ der Patientin. Die offenkundige Fehlleistung und mein erstes Nicht-Verstehen erklären nicht, auf welchem Wege mich die Empfindungen erreicht haben, die ich als fremd wahrnahm. Ich gehe im Verstehensprozess der Stunde davon aus, dass es auf vollkommen unbewusstem Weg geschah. Die Fremdheit ist ein klinisches Zeichen, dem ich immer wieder begegne (Dreyer 2017). Es handelt sich um eine besondere Qualität des Fremden, Unerklärlichen und Ich-Dystonen. Zunächst gilt es, meine eigenen Affekte in ihrer Bedeutung für die Behandlung zu erschließen. Dieser Weg führt so meine Erfahrung - zum Erfolg, wenn ich mich dazu entschließe, transparent zu benennen, dass es da etwas gibt, das ich spüre, das ich mir allein aber nicht erklären kann. Dieses Vorgehen gründet in der Annahme, dass es sich um eine introjektive Identifizierung handelt, durch die ich am Unbewussten meines Patienten oder Analysanden unmittelbar teilhabe und die über meine Gegenübertragung aufzufinden ist. Der Austausch darüber schafft Bewusstsein, wenn er in einer Atmosphäre der Transparenz und Teilhabe stattfindet. Es ist weiter meine These, dass es sich um Abläufe handelt, die regelhaft und in verschiedenen Analysen ähnlich wiederkehren.

Um dies näher auszuführen, werde ich nun unter dem Aspekt von Transparenz und Teilhabe die Theorie zur Gegenübertragung und zur introjektiven Identifizierung näher betrachten, um danach - erneut an meinem Fall - meine Thesen abschließend zu diskutieren.

\section{Gegenübertragung}

Bahnbrechend für unser heutiges Verständnis der Gegenübertragung waren die Veröffentlichungen von Heimann (1950/2016a, S. 111-117) „Zur Gegenübertragung“ und Heinrich Racker (1950/2002) „Übertragung und Gegenübertragung - Studien 
zur psychoanalytischen Technik“. Beide Arbeiten erscheinen im gleichen Jahr und betonen unabhängig voneinander, dass die Gegenübertragung nicht länger als Hindernis zu betrachten ist, sondern vielmehr ein förderndes Element für die Analyse darstellt. Das, was zunächst als technischer Fehler erschien, wurde zum Katalysator des therapeutischen Prozesses. Racker (1950/2002, S. 150-151) sah bereits die Möglichkeit der wechselseitigen Bezogenheit von Übertragung und Gegenübertragung, von Analytiker und Analysand. Es kann also auch die (Gegen-)Übertragung des Analytikers sein, die erst die Übertragung des Analysanden anschiebt, die dann anschließend vom Analytiker gedeutet wird. Das Henne-Ei-Problem löst sich auf, wenn man nicht mehr in Vorher-nachher-Kategorien denkt, sondern von einer Wechselwirkung ausgeht. Der Satz ,die Gegenübertragung des Analytikers geht der Übertragung des Patienten voraus“, drückt dann nur die eine Hälfte des Geschehens aus. Er könnte ergänzt werden durch den Satz: „Die Übertragung des Analytikers geht der Gegenübertragung des Analysanden voraus“.

Heimann (2016b) vertieft ihre Auffassung 30 Jahre nach der Erstveröffentlichung, 1978, mit einem Titel, der bereits die Kernaussage der Arbeit formuliert: „Über die Notwendigkeit für den Analytiker, mit seinem Patienten natürlich zu sein“. Sie berichtet eine überraschende „Intuitivdeutung“, die, wäre sie nicht so treffend, kränkend gewirkt hätte. Zur Überraschung beider öffnet die Analytikerin durch diese Deutung eine lange von ihrer Patientin verschwiegene Geschichte, die die Analyse entscheidend voranbringt. Die Autorin weist darauf hin, dass ihr Vorgehen weiterer Ausarbeitung bedürfe.

Racker und Heimann machten mit ihren Veröffentlichungen deutlich, welch überraschende Erkenntnisse die Analyse der Gegenübertragung zugänglich macht. Andere Autoren entwickeln die intersubjektive Auffassung von Gegenübertragung weiter: Jacobs (1986), Gabbard (1995) und Ogden (2006), bezogen auf das Feldkonzept schließlich Baranger und Baranger (2008) sowie Ferro (2005). Auf eindrucksvolle Weise deutet besonders Ogden (2006, S. 61) diese Ereignisse in Analysen. Er zeigt, wie ,enactments“ oftmals für das Verständnis des unbewussten Prozesses entscheidend sind und die Aufgabe darin besteht, sich ihnen träumerisch überlassen zu können, bis sich ein neues Verständnis eingestellt hat: Ogden beschreibt damit eine radikale Verwandlung.

„Was dabei im psychoanalytischen Prozess geschieht, ist ein partieller Zerfall der unbewussten Dialektik von Subjektivität und Intersubjektivität. Dieser Zerfall führt schließlich zur Erzeugung einer besonderen Form des analytischen Dritten, der sich Analytiker und Analysand als getrennte Subjekte in hohem Maße unterwerfen (dem ,Dritten der Unterwerfung'). Eine erfolgreiche Psychoanalyse bedeutet, dass die daran Beteiligten dieses unbewusste Dritte, das nur eine Spielart von Intersubjektivität ist, aufheben und sich den im psychoanalytischen Prozess freilich verwandelten Subjektstatus wieder aneignen, indem sie zu eigenständigen (und gleichwohl voneinander abhängigen) Individuen werden. Das erreichen sie dadurch, dass sie einander gegenseitig anerkennen."

Wie gelangen die von Racker theoretisch ausgearbeiteten und von Ogden klinisch dargestellten Phänomene in die Interaktion der Partner der Dyade, und wie können sie bewusst gemacht werden? Eine Antwort ergibt sich aus der Beschreibung der Brückenfunktion der introjektiven Identifizierung. 


\section{Die introjektive Identifizierung, ein Brückenkonzept}

Ogden beschreibt klinisch den sich im Unbewussten abspielenden Brückenschlag zwischen den beiden an der analytischen Situation Beteiligten, unterscheidet jedoch nicht zwischen projektiver und introjektiver Identifizierung. Er spricht nur von projektiver Identifizierung. Ich halte es für zulässig, seine Beschreibung einer partiellen und passageren Auflösung der Subjektgrenzen für die introjektive Identifizierung zu übernehmen: Gerade in der introjektiven Identifizierung sind die Übernahme und Vermischung von Affekten beider beteiligter Subjekte zu beobachten. Bollas (1997, S. $168 \mathrm{ff}$.) fügt den beiden Mechanismen noch die extraktive Introjektion hinzu.

Zunächst ist eine Abgrenzung der introjektiven von der projektiven Identifizierung erforderlich. In der Literatur werden beide Vorgänge in der großen Übersichtsarbeit von Grotstein (1994) beschrieben. Sodré (2012) und Spillius (2012) unterscheiden zwischen projektiver und introjektiver Identifizierung: Sie betonen die positivlibidinösen Seiten der introjektiven Identifizierung und warnen zugleich vor ihrer Idealisierung. O'Shaugnessy (2007, S. 105) weist darauf hin, dass die introjektive Identifizierung im Vergleich zur projektiven Identifizierung insgesamt wenig beachtet wurde. Dies ergänzt Scharff (1992) sowohl für die projektive als auch für die introjektive Identifizierung aus intersubjektiver Sicht. Eekhoff (2016) benennt schließlich die außergewöhnliche Qualität des wechselseitigen Austauschs von unbewusst zu unbewusst und nennt die Wirkung der introjektiven Identifizierung in ihrem Titel „evokativ“.

Eine Differenzierung zwischen den unbewussten Vorgängen der projektiven und der introjektiven Identifizierung:

Bei der projektiven Identifizierung wird der projizierte Inhalt vom Projizierenden als nicht mehr zu seiner Person gehörend wahrgenommen, der projizierte Inhalt wird stattdessen demjenigen zugerechnet, der damit projektiv identifiziert wurde; der Projizierende ist dadurch vom Inhalt seiner Projektion befreit.

Bei der introjektiven Identifizierung dagegen bleibt der innere Dialog des Introjizierenden mit den introjizierten Inhalten bestehen. Introjiziert wird etwas Fremdes, das in Teilen als fremd erkennbar bleiben kann, in anderen Teilen dem Erfahrungshorizont des Introjizierenden nahe, vertraut ist. Letzteres bildet den Anker für die Bewusstwerdung.

Die extraktive Introjektion (Bollas 1997, S. 173) ist ein Mechanismus, „bei dem ein Mensch in das Innenleben eines anderen eindringt und bestimmte Elemente davon vereinnahmt. Das Opfer einer extraktiven Introjektion fühlt sich der betreffenden Anteile seines Selbst beraubt. Ist das Opfer ein Kind, so hat es keine klare Vorstellung davon, weshalb es auf bestimmte Elemente seines psychischen Lebens kein Recht zu haben scheint.“ Sie spielt sich also im Inneren des Introjizierenden $\mathrm{ab}$ und steht der projektiven Identifizierung in ihrem aggressiv-aufgeladenen Modus nahe.

Wie wird aus einer introjektiven Identifizierung ein fruchtbarer Beitrag zur Behandlung? Die Brückenbildung der introjektiven Identifizierung beruht auf der zweiseitigen Eigenschaft des introjizierten Inhalts: Einerseits bleibt er spürbar behaftet mit etwas Fremdem, bislang Unbekanntem, und andererseits ist er stabil eingebettet in die Psyche dessen, der introjiziert. Mit etwas Übung kann dieser Doppelcharakter 
vom Analytiker im Dialog herausgespürt werden. Es findet zuerst eine innere Prüfung und Aufarbeitung in der Phase der Gegenübertragungsanalyse im Analytiker statt: Was wurde mir zur Introjektion angeboten, und was wurde von mir introjiziert? Soweit spielt sich der Vorgang im Inneren des Analytikers ab.

Im nächsten Schritt wird mithilfe der noch vagen Hypothese ein Brückenschlag versucht; eingeleitet durch Worte, die vorsichtig das Ungewöhnlich-Unsichere des Vorgehens zum Ausdruck bringen, um dem Analytiker einen Rückzug von seiner Idee zu ermöglichen, sollte ein Irrtum vorliegen. Sollte der Patient durch neu ins Bewusstsein tretende Einfälle dem Dialog eine entscheidende Wendung geben, dürfen wir davon ausgehen, dass es gelungen ist, unbewusstes und abgespaltenes Material aufzuspüren. Wir prüfen und präzisieren dann unsere Hypothese. Wenn dies alles gelingt, ist die Analyse einen wesentlichen Schritt vorankommen.

Hier noch einmal die möglichen Wirkungen und Chancen der Konzeptualisierung der introjektiven Identifizierung als einem Brückenkonzept:

- Dem Analytiker wird es möglich, Empfindungen aus seinem eigenen Unbewussten mit Anteilen aus dem Unbewussten seines Analysanden zu verbinden, sich diese bewusst zu machen und als analytisches Material zu nutzen: eine Bereicherung des analytischen Prozesses.

- Im Analysanden bleiben die Prozesse der introjektiven Identifizierung zunächst unbewusst, sie stammen aus seiner Kindheit und bedürfen der analytischen Aufarbeitung, da sie mit umfangreichen pathologischen Abwehrvorgängen verbunden sind. Mithilfe der inneren Arbeit des Analytikers gelingt es, einen bewussten $\mathrm{Zu}$ gang zu finden und die Gründe für die Abspaltung ins Unbewusste aufzuheben.

Im Konzept der introjektiven Identifizierung ist eine Entwicklungsmöglichkeit von der Pathogenese zur Salutogenese angelegt, die ihre Wurzeln in der frühen Mutter-Kind-Interaktion hat (hierzu Bion 2009; Fonagy und Target 2006; diskutiert bei Dreyer 2017, S. 117 ff., S. 174). Ergänzen möchte ich an dieser Stelle noch, dass es durchaus nicht selten vorkommt, dass die Schritte der Bewusstwerdung vor allem auf der Seite des Patienten ablaufen.

\section{Der Fall, Biografie, Psychodynamik, Behandlung des Traumas}

Die 30-jähige Patientin stammt aus einer kleinen Stadt im Alentejo, Portugal. Da ich kein Portugiesisch kann, weichen wir bisweilen ins ,neutrale“ Französisch aus; ihres ist besser als meines. Manchmal verständigen wir uns auf Englisch; in der Zeit der Behandlung wird ihr Deutsch immer besser.

Sie wurde als Teenager von ihrem Klavierlehrer missbraucht. Inzwischen ist er angezeigt und verurteilt; die Aussage meiner Patientin spielt dabei eine wesentliche Rolle. Ihr Vater wurde von seinem Vater körperlich misshandelt, kann bis heute nicht darüber sprechen und ist körperlich und seelisch krank.

Ich finde zu der folgenden psychodynamischen Hypothese: Die Patientin, Einzelkind, hat in einer fatalen Mischung aus Einfühlung und Identifikation mit dem Vater aktiv dessen Kindheitssituation reproduziert, sein Kreuz (Grotstein 1994) auf sich genommen, um sich auf diesem Weg doch noch in den ansonsten unnahbaren Vater 
einfühlen zu lernen: Auf diesem Boden kann sich später der Missbrauch durch den Klavierlehrer entwickeln.

Als die Patientin den Klavierunterricht bei diesem Lehrer bereits beendet hatte, erzählt ihr die Schwester des Vaters, die jahrelang quer durch Europa auf der Flucht vor ihrer Familie war, von der schlimmen Kindheit des Vaters und bestätigt damit, was sich die Patientin emotional bereits erschlossen hatte. Ein Gespräch mit ihm darüber ist bis heute nicht möglich. Auch im Leben der Patientin folgen wilde Jahre des inneren und äußeren Kampfes mit Gothic-Phase, Selbstschädigung und Beziehungslosigkeit. Dessen ungeachtet vervollständigt sie ihr Klavierstudium in Mailand, Paris und London, zuletzt an einer Musikhochschule in Deutschland. Sie ist heute selbst Klavierlehrerin und leidet unter den Folgen ihrer Erfahrungen in ihrem Unterricht.

$\mathrm{Zu}$ ihrer Missbrauchserfahrung: Sie berichtet über Unterrichtsbegegnungen im Haus des Klavierlehrers, fernab anderer Häuser, wo er ihr seine Hände auf die Schultern gelegt und sie aufgefordert hat, ihr T-Shirt auszuziehen. Er sagte, ,man muss als Musiker nackt spielen können“. Ein andermal setzt er sich mit geöffneten Beinen vor sie hin, weist auf sein Glied und fragt, ob sie wisse, warum er das tue. Im Prozess wird offenbar, dass es viele derart von ihm missbrauchte Klavierschülerinnen gibt; sie war die Älteste und Erste, die Anzeige erstattet hat. Ihre Eltern ahnten nichts, und sie verbarg es vor ihnen.

In den zwei Jahren der zweistündigen Behandlung im Sitzen gibt es viele bewegende Momente, in denen beispielsweise meine Art, die Treppe herunterzukommen, sie an ihren ,professeur“ erinnert; dazu unsere Mikrointeraktion: Im ersten Behandlungsjahr bemerkt sie einmal, sie könne nicht denken. Mir geht es in der Stunde ähnlich. Schließlich kommt mir der Einfall: „Es ist die Zerstörung durch den Missbrauch: Der Missbrauch zerstört das Denken. Wenn ich als ,Professeur ' die Treppe herunterkomme, haben wir jetzt hier, was sonst draußen bleibt: die Erinnerung an den Missbrauch." Ab diesem Moment verbindet sich mit meinem Treppab die alte Missbrauchssituation.

Die nun folgende, ausführliche Sequenz liegt zwei Wochen vor dem Material meiner Eingangsvignette. Die Patientin kommt zurückhaltender als sonst in eine Stunde. „Was ist, wenn wir schweigen?“ fragt sie mich. Ich vermute, was sie bestätigt, dass sie darüber nachdenkt, ob sie mich dann langweilen würde. Das wäre eine große Gefahr, denn in ihrer Fantasie wird aus Langeweile Missbrauch. Wir gelangen durch diese unscheinbare Eingangsfrage unmittelbar in die vergangene Welt ihres Missbrauchs.

Bald darauf fasst sie sich versehentlich mit dem Finger in ihr rechtes Auge und sagt „Entschuldigung“. Mich berührt schmerzlich, dass sie sich selbst Schmerzen zufügt und sich auch noch dafür entschuldigt - unklar bleibt, bei wem. Wir sprechen weiter darüber, was es mit dem Schweigen auf sich haben könnte, und dass dies für sie mit der Angst einhergeht, es könnte so etwas Schreckliches wie ein Missbrauch passieren. Mir dämmert, was ich noch nicht glaube, aussprechen zu können: Meine Assoziation, ein Auge auf jemanden werfen, führt mich schließlich zu der Deutung: Ob sie sich wohl deshalb entschuldigt habe, weil sie - so seltsam es auch klingen mag - tatsächlich Schuld empfinde. Schuld dafür, dass sie „Langeweile“ habe entstehen lassen, wodurch sie den Professeur provoziert haben könnte. Oder 
genauer: Sie könnte im Rückblick denken, dass sie ihn provoziert und dadurch das ganze Missbrauchsunglück selbst ins Rollen gebracht habe. Die Patientin denkt kurz darüber nach; ich bin sehr in Sorge, ob ich ihr zu viel zugemutet habe. Dann lacht sie befreit und sagt „Das stimmt!“ Sie schüttelt den Kopf darüber, was ich herausgefunden habe. Sie ist durch meine Deutung nicht gekränkt, sondern befreit. Ein besonderer Moment in der Behandlung.

In eine der Folgestunden kommt sie in guter Stimmung, setzt sich und lacht: Es sei schön gewesen im Konzert, das sie im Ausland gespielt hat: Über Nacht sei sie mit drei Kollegen, ,zwei Violinen und einem Cello“ hingefahren. Kleine Stadt, Kirche mitten im See auf einer Insel, die aus dem See herausragt. Es hat gut geklappt. Morgen will ihr Chef mit ihr sprechen, alle waren sehr angetan, bei ihr lief es sehr gut. Auch die anderen finden das. Überhaupt ist sie frei im Rücken, keine Verspannungen mehr. Ihre Kollegen sind oft schlechter Laune, sie nicht. Nach einiger Zeit, in der ich darüber nachdenke, welche Bedeutung wohl dieses Bild des Sees mit Insel und Kirche hat, fragt mich die Patientin, woran ich denke. Ich spreche in meiner Antwort über meine im Stillen entwickelte Hypothese: Ob es wohl richtig sei, von einer Art Umkehrung ins Gegenteil zu sprechen: Da ist die Insel im See, umgeben von Wasser, eine isolierte Idylle mit Konzert und Besuchern, und als Bild des Gegensatzes dazu das Haus des Professeur, einsam und isoliert, isoliert im Schrecken! Zu meiner Überraschung antwortet die Patientin: „Ja, genau, das ist es!“ Ich bin perplex, denke, das ist ihr schnelles Tempo, und weiß selbst nicht, was ihre Antwort bedeutet. Ich hatte unfertige Gedanken ausgesprochen und finde zunächst keine Verbindung zu ihrer Reaktion. „Ich weiß jetzt nicht, wie Sie das meinen“, sage ich deshalb. Daraufhin erläutert sie mir, wie sie in den letzten Tagen immer wieder über einen Satz von mir nachdenken muss, der sie jedes Mal aufs Neue befreie. Es war meine Antwort auf die Frage des Professeur, die sie so sehr quälte: Er fragte sie, ob sie Sex hatte. „He, mit 13!?““, stellt sie ihre hochgradige Irritation von damals dar, hinter der ich die extraktive Introjektion des Klavierlehrers vermute. Befreit habe sie, dass ich vor ein paar Stunden kommentiert hatte: „Das war sein Gedanke!“ Seither wird sie nicht mehr von Fragen gequält, weil sie erkennen kann, dass es gar nicht ihr Thema, sondern seines war. Offenbar wurden die Folgen der extraktiven Introjektion durch den Professor, der ihr das Gefühl ihrer unschuldigen Körperlichkeit genommen hatte, durch meinen Hinweis, dass es seine Gedanken waren, wirksam aufgelöst! Immer wieder sei sie seit unserer Stunde auf diese Erkenntnis zurückgekehrt, und jedes Mal sei danach die Geschichte anders, befreit verlaufen! Das habe ihr gute Laune gemacht, dass sie sich nicht mehr durch die Frage ,Was hat ihn zu der Vermutung gebracht?" belastet fühle.

Ich glaube verstanden zu haben und sage: „Ich habe S/sie Ihnen geschenkt.“ Gleich ist mir meine Bemerkung in ihrer Doppeldeutigkeit peinlich: Einerseits kann „sie“ auf die gute Laune bezogen werden, andererseits kann „Sie“ auf die Patientin als Person bezogen werden, und das eine oder das andere hätte ich ihr ,geschenkt“. Die Patientin befreit mich aus meiner peinlichen Lage und verbessert mich: „Nein, zurückgegeben!“‘ Ich antworte erleichtert: „Ja, Sie haben recht! Zurückgeben ist das richtige Wort.“ Ich kann noch anfügen: „Hier hat sich zwischen uns eben wiederholt, worüber wir sprachen - in einer kleineren Dimension natürlich!“ Dann ist die Stunde zu Ende. 
Zwei Wochen später ereignet sich die Stunde, von der ich eingangs berichtet habe: Meine Patientin versucht, die Praxistüre mit ihrem Schlüssel aufzuschließen, und mich beschleicht das Gefühl der Traurigkeit.

\section{Zusammenfassende Diskussion}

In psychoanalytischen Behandlungen sind viele Wege möglich, auf denen Unbewusstes verstanden werden kann. Nur einer davon wird verwirklicht. Im Prozessgeschehen stellt sich die Frage: Wie lange - im Hinblick auf das Ziel der Bewusstwerdung - soll man in Indifferenz und Offenheit abwarten und sich dem Wachtraumdenken (Bion 1962) überlassen? Wann und wie lassen sich introjektive Prozesse optimal verstehen? Es kann im Zuhören zu früh gedeutet oder zu lange gewartet und in beiden Fällen die Besonderheit des Augenblicks verpasst werden. Die Bedeutung des Augenblicks für eine Deutung fasst Strachey (1934, S. 150) in den Begriff des ,point of urgency“. Für die Darstellung in einer Fallvignette und ganz generell gilt darüber hinaus: Die Plausibilität einer Fallgeschichte bleibt gebunden an idiosynkratische Kreisprozesse, deren Ablauf sich im Kreislauf zwischen Annahme einer Psychodynamik, Deutung der Interaktion, Beobachtung der Reaktion, Vermutung über erreichte Effekte und Korrektur der Annahme abspielt. Jede prognostische Aussage bleibt ebenso einem Kreisprozess verhaftet und immer offen für alternative Konzeptualisierungen und alternative Kreisprozesse.

Zur wissenschaftstheoretischen Einordnung dieses Vorgehens greife ich auf die Erläuterungen zum „Denken in Fällen“ von Hampe und Strassberg (2015, S. 281; vgl. obigen Abschnitt „Eine Fallvignette“) zurück: Durch Generalisierung, wie sie uns auch aus Mythen und Geschichten entgegentritt, entstehen „Entwicklungsschemata" und Narrative, die einen gesetzmäßigen Charakter haben. Im Alltag der klinischen Stunde wird das „Entwicklungsschema“ zur Grundlage der deutenden analytischen Aktivität. Gleichzeitig damit bleibt auch das ,Entwicklungsschema“ selbst offen für Modifikation. Mit Schülein (2002) ist davon auszugehen, dass konnotative Theorien wie die Psychoanalyse zwangsläufig offen und unscharf bleiben. Falldarstellungen beziehen ihre Qualität aus der Plausibilität ihrer Darstellung; eine Objektivität der Falldarstellung gibt es nicht.

Dies habe ich theoretisch und klinisch zu zeigen versucht: Die Annahme der introjektiven Identifizierung als interaktiver Abwehr- und Übertragungsmechanismus öffnet dann ein neues Verständnis für Elemente des psychoanalytischen Prozesses, wenn in einer Haltung von Transparenz und Teilhabe vorgegangen wird. Behandlungstechnisch trägt die vorübergehende Verwirrung zur Schwierigkeit des Analysierens bei, die auf dem Weg entsteht, den Ogden beschreibt. Auch Eekhoff (2016) weist eindrücklich auf die Schwierigkeiten hin.

Nun zur Diskussion des dargestellten Behandlungsverlaufs: Im zeitlich ersten Behandlungsabschnitt, den ich als zweiten berichte, geht es um Zuhören in einer Haltung von Reverie und Wachtraumdenken, wie es Ferro (2006) als bedeutende Entdeckung Bions hervorgehoben hat. Im dargestellten Abschnitt fasst sich meine Patientin in ihr eigenes Auge und entschuldigt sich sofort dafür. Die kleine Selbstverletzung mit Entschuldigung kann als symbolische Darstellung ihres aktiv-fanta- 
sierten Handlungsanteils am Missbrauch verstanden werden. Meine Deutung dieses Zusammenhangs führt in der Stunde zum Bericht über eine befreit-schöne Reise in die Welt der Musik. Die heitere, helle Idylle nimmt eine Stimmung vorweg, in der die Patientin dann zwei Wochen später in die Stunde kommt, die ich ausführlich darstelle.

$\mathrm{Zu}$ Beginn dieser letzten Stunde eines dreiwöchigen Behandlungsabschnitts bleibt mir meine Traurigkeit lange fremd, die in auffälligem Gegensatz zur heiteren Stimmung meiner Patientin steht. Ich stelle mir in der Analyse meiner Gegenübertragung Fragen: Bin ich traurig darüber, dass sie sich trennt, und nehme ich die Gefühle des verlassenen Freundes vorweg? Könnte die Ankündigung der Patientin, dass sie sich von ihrem Freund trennt, ein Menetekel dafür sein, dass sie sich bald auch von mir trennt? Zahlreiche Interpretationen sind an dieser Stelle vorstellbar.

Der weitere Verlauf der Stunde führt in eine überraschend andere Richtung, die von den kleinen Zeichen des Misslingens zu Beginn der Stunde ausgeht: Die Praxistüre öffnet sich mit dem Schlüssel der Patientin nicht, und meine erste Deutung öffnet keinen Weg zu ihrem Unbewussten. Ich spüre deutlich die mir unerklärliche Traurigkeit und erlebe bewusst eine Diskontinuität zur Heiterkeit meiner Patientin. Ich habe gelernt, auf solche diskontinuierlichen Momente (Dreyer 2017, S. 93 ff.) besonders zu achten, weil sie Vorboten für noch unbewusstes Material sein können. Ich weiß, dass sich diese Diskrepanz am ehesten dadurch auflösen lässt, dass ich mein Empfinden mitteile. Ich habe vielfach erfahren, wie ein solches Vorgehen dazu beiträgt, eine Spaltung zwischen den Personen (hier die Traurigkeit des Analytikers, da die Heiterkeit der Analysandin) und innerhalb der Affekte der Beteiligten (mein Gefühl der Fremdheit gegenüber meinem Affekt der Traurigkeit) aufzuheben.

Auf meine Bemerkung zur Diskrepanz in mir und zwischen uns antwortet die Patientin überraschend und sagt: „Ich bin dumm“. Sie erläutert ihre Empfindung mit einem literarischen Bild, das mir die Selbstverdummung als Schutzmechanismus erklärt. Das legt mir den Gedanken nahe, dass ich Gefühle von Traurigkeit über die Auswirkungen des Missbrauchs empfinde, die $i h r$ noch unbewusst sind. Ich gelange so zur Annahme einer introjektiven Identifizierung: Mein mir fremdes Gefühl von Traurigkeit enthält die von mir introjizierten Affekte. Ich war eindeutig mit diesem Gefühl identifiziert; es war „mein“ Gefühl, über dessen Ursprung ich mir Gedanken machte, und zugleich war es dem (noch unbewussten) Empfinden meiner Patientin nahe.

Die Deutung des Zusammenhangs, Selbstverdummung als Schutz vor den Auswirkungen des traumatischen Geschehens, wirkt befreiend; meine Patientin findet ihre Traurigkeit wieder. Die Traurigkeit, die ich zu Stundenbeginn diskontinuierlich empfinde, kann als eine Vorwegnahme, eine Vorleistung des Analytikers in Gestalt einer introjektiven Identifizierung verstanden werden. Loch versteht unter einer „notwendigen Vorleistung“ (1965, S. 20/21) den Moment, in dem der Analytiker den infantilen Übertragungswunsch seiner Analysanden zunächst in seiner Gegenübertragung auflöst. Dann kann auch der Analysand erkennen, was er übertragen hat.

In meiner Fallvignette entdeckt die Patientin, dass sie weder beim kranken, misshandelten Vater noch beim aggressiv-missbrauchenden Klavierlehrer sitzt. Lochs Gedankengang wirkt sich rückblickend konzeptualisierend aus. Thomä betont in 
seiner Arbeit aus dem Jahr 1981 (... Vom spiegelnden zum aktiven Analytiker) die Gleichzeitigkeit von Handeln und Reflexion; zwei Arbeiten, die wesentlich zur Entwicklung der intersubjektiven Theorie in Deutschland beigetragen haben (1981).

In das affektive Geschehen ist der Analytiker - wie ich in meinem Fallbeispiel zeige - unmittelbar verwickelt (Ogden 2006, S. 61). Durch die unbewusste Übernahme der Affekte verschwimmen die Subjektgrenzen. Zuerst übernimmt der Analytiker das Misslingen, dann formuliert er sein ihm unerklärliches Gefühl und öffnet damit den Weg zum Verständnis der introjektiven Identifizierung: Auch die Patientin gewinnt ihre Sprache wieder und spricht von Selbstverdummung - die Auflösung der introjektiven Identifizierung gelingt.

Theoretisch stehen die hier dargestellten klinischen Beobachtungen im Einklang mit Ferenczis Verständnis der Ich-Einschränkung (1933). Um verstehen zu können, braucht der Analytiker genügend Negative capability (Bion 1970, S. 125); er muss in Ogdens Formulierung „Unter-das-Joch-Gehen“ können (engl. ,,subjugate“; Ogden 2004, S. 189, Hervorhebung K.-A. D.): ,the individuals engaged in this form of relatedness [projective identification] unconsciously subjugate themselves to a mutually generated intersubjective third .... In projective identification, analyst and analysand are both limited and enriched“".

Der auf den ersten Blick vielleicht vage und unsicher erscheinende Verstehenszusammenhang gewinnt wachsende Plausibilität, wenn wir bereit sind, introjektividentifizierende Mechanismen auf uns wirken zu lassen. Die Prüfung unserer psychodynamischen Hypothesen erfolgt danach im Querschnitt des klinischen Augenblicks und im Längsschnitt des analytischen Prozesses, der Biografie und der äußeren Ereignisse.

Die therapeutisch-psychoanalytische Arbeit in einer Haltung von Transparenz und Teilhabe ist zunächst keine Selbstverständlichkeit. Der Verstehensweg über die introjektive Identifizierung als bedeutungsvollem Abwehr- und Interaktionsmechanismus setzt Verständnis und Erfahrung in der Haltung von Transparenz und Teilhabe voraus. Die Arbeit mit der introjektiven Identifizierung eröffnet für die klinische Arbeit wertvolle Perspektiven. Transparenz fördert die selbstanalytischen Fähigkeiten unserer Patienten. Transparenz und Teilhabe gemeinsam schärfen den Blick für die komplexen Abwehr- und Interaktionsmechanismen der introjektiven Identifizierung im psychoanalytischen Prozess.

Interessenkonflikt K.-A. Dreyer gibt an, dass kein Interessenkonflikt besteht.

\section{Literatur}

Altmeyer M, Thomä H (Hrsg) (2006) Die vernetzte Seele: Die intersubjektive Wende in der Psychoanalyse. Klett-Cotta, Stuttgart

Baranger M, Baranger W (2008) The analytic situation as a dynamic field. Int J Psychoanal 89(4):795-826 Bion WR (1962) Learning from experience. Heinemann, London

Bion WR (1970) Attention and interpretation. London, Tavistock

Bion WR (2009) Raster und Zäsur: Zwei Abhandlungen. Brandes \& Apsel, Frankfurt am Main

Bohleber W (2014) Gesellschaftliche Demokratisierung und der Aufstieg des intersubjektiven Paradigmas in der Psychoanalyse: Eine kritische Bestandsaufnahme. In: Janta B, Waltz-Pawlita S, Unruh B (Hrsg) Unzeitgemäßes. Psychosozial-Verlag, Gießen, S 53-71 
Böhme I (2014) Die Wissenschaft von der Begegnung der Subjekte in kleinianischer und bionianischer Perspektive. In: Potthoff P, Wollnik S (Hrsg) Die Begegnung der Subjekte: Die intersubjektiv-relationale Perspektive in Psychoanalyse und Psychotherapie. Psychosozial-Verlag, Gießen, S 79-97

Bollas C (1997) Der Schatten des Objekts: Das ungedachte Bekannte: Zur Psychoanalyse der frühen Entwicklung. Klett-Cotta, Stuttgart

Cremerius J (1984) Die psychoanalytische Abstinenzregel. Vom regelhaften zum operationalen Gebrauch. Psyche - Z Psychoanal 38(9):769-800

Dreyer K-A (2017) Transparenz und Teilhabe. Veränderungen in der psychoanalytischen und psychodynamischen Behandlungstechnik. Psychosozial-Verlag, Gießen

Eekhoff JK (2016) Introjective identification: the analytic work of evocation. Am J Psychoanal 97:989-1003

Ermann M (2014) Der Andere in der Psychoanalyse: Die intersubjektive Wende. Kohlhammer, Stuttgart

Ferenczi S (1933) Sprachverwirrung zwischen den Erwachsenen und dem Kind. In: Balint M (Hrsg) Schriften zur Psychoanalyse, Bd. 2. Fischer, Frankfurt am Main, S 303-313

Ferro A (2005) Im analytischen Raum: Emotionen, Erzählungen, Transformationen. Psychosozial-Verlag, Gießen

Ferro A (2006) Clinical implications of Bion's thought. Int J Psychoanal 87:989-1003

Freud S (1912e) Ratschläge für den Arzt bei der psychoanalytischen Behandlung. GW VIII, S 376-387

Freud S (1915b) Triebe und Triebschicksale. GW X, S 209-232

Freud S (1919a) Wege der psychoanalytischen Therapie. GW XII, S 183-194

Freud S (1923b) Das Ich und das Es. GW XIII, S 235-289

Gabbard GO (1995) Countertransference: The emerging common ground. Int J Psychoanal 76(3):475-485

Grotstein JS (1994) Projective Identification Reappraised-Part I: Projective Identification, Introjective Identification, the Transference/countertransference Neurosis/psychosis, and their Consummate Expression in the Crucifixion, the Pieta, and "Therapeutic Exorcism". Contemp Psychoanal 30:708-746

Hampe M, Strassberg D (2015) Nicht vereinheitlichte Wissenschaftstheorie und Psychoanalyse. In: Allert G, Rühling K, Zwiebel R (Hrsg) Frühjahrstagung 2015 Deutsche Psychoanalytische Vereinigung. Psychosozial-Verlag, Gießen, S 265-281

Heimann P (2016a) Zur Gegenübertragung. Gegenübertragung und andere Schriften zur Psychoanalyse. Vorträge und Aufsätze aus den Jahren 1942-1980. Klett-Cotta, Stuttgart, S 111-117

Heimann P (2016b) Über die Notwendigkeit für den Analytiker mit seinem Patienten natürlich zu sein. Gegenübertragung und andere Schriften zur Psychoanalyse. Vorträge und Aufsätze aus den Jahren 1942-1980. Klett-Cotta, Stuttgart, S 416-429

Jacobs TJ (1986) On Countertransference Enactments. J Am Psychoanal Assoc 34:289-307

Klein M (1946) Bemerkungen über einige schizoide Mechanismen. In: Klein M (Hrsg) Gesammelte Schriften. I/1. frommann-holzboog, Stuttgart, S 347-368

Loch W (1965) Übertragung - Gegenübertragung. Psyche Z Psychoanal 19(1):1-23

Lorenzer A (1970) Sprachzerstörung und Rekonstruktion. Suhrkamp taschenbuch wissenschaft 31. Suhrkamp, Frankfurt/Main

Luiz C (2006) Pushing through boundaries of inner space: the need for analytic transparency in the treatment of a juggler. Mod Psychoanal 31(2):151-181

Mertens W (2013) Das Zwei-Personen-Unbewusste - unbewusste Wahrnehmungsprozesse in der analytischen Situation. Psyche Z Psychoanal 67(9):817-843

Ogden MD (2004) The analytic third: implications for psychoanalytic theory and technique. Psychoanal Q 73(1):167-195

Ogden TH (2006) Das analytische Dritte, das intersubjektive Subjekt der Analyse und das Konzept der projektiven Identifizierung. In: Altmeyer M, Thomä H (Hrsg) Die vernetzte Seele: Die intersubjektive Wende in der Psychoanalyse. Klett-Cotta, Stuttgart, S 35-64

Orwell G (2002) 1984. Heyne, München

O'Shaughnessy E (2007) Introjektive und projektive Identifizierung und ihre Auswirkungen auf das Ich. In: Frank C, Weiß H (Hrsg) Projektive Identifizierung: Ein Schlüsselkonzept der psychoanalytischen Therapie. Klett-Cotta, Stuttgart, S 88-107

Racker H (2002) Übertragung und Gegenübertragung - Studien zur psychoanalytischen Technik, 6. Aufl. Ernst Reinhardt, München, Basel

Scharff JS (1992) Projective and Introjective identification and the use of the therapist's self. Rowman \& Littlefield Publishers, Lanham

Schoenhals Hart H (2007) Zur Begriffsverwirrung bei der projektiven und introjektiven Identifizierung. In: Frank C, Weiß H (Hrsg) Projektive Identifizierung: Ein Schlüsselkonzept der psychoanalytischen Therapie. Klett-Cotta, Stuttgart, S 155-179 
Schülein JA (2002) Autopoietische Realität und konnotative Theorie: Über Balanceprobleme sozialwissenschaftlichen Erkennens. Velbrück Wissenschaft

Sodré I (2012) Who's who? - Notes on pathological identifications. In: Spillius E, O'Shaughnessy E (Hrsg) Projective identification - the fate of a concept. Routledge, London, New York, S 55-60

Spillius E (2012) Developments by British Kleinian analysts. In: Spillius E, O’Shaughnessy E (Hrsg) Projective identification - the fate of a concept. Routledge, London, New York, S 30-33

Stern DN (2010) Der Gegenwartsmoment: Veränderungsprozesse in Psychoanalyse, Psychotherapie und Alltag. Brandes \& Apsel, Frankfurt am Main

Stolorow RD, Atwood GE (1992) Contexts of being: the Intersubjective foundations of psychological life Bd. 12. The Analytic Press, Hillsdale

Strachey J (1934) The nature of the therapeutic action of psycho-analysis. Int J Psychoanal 15:127-159

Target FPM (2006) Psychoanalyse und die Psychopathologie der Entwicklung. Klett-Cotta,, Stuttgart

Thomä H (1981) Schriften zur Praxis der Psychoanalyse: Vom spiegelnden zum aktiven Psychoanalytiker. Suhrkamp, Frankfurt am Main

Dr. med. Karl-Albrecht Dreyer Facharzt für Psychiatrie, psychosomatische Medizin, Psychotherapie, Psychoanalyse (DPV, DGPT). Dozent, Supervisor und Lehranalytiker der Psychoanalytischen Arbeitsgemeinschaft Ulm. Wissenschaftliche Schwerpunkte: Frequenzfragen mit Herausgabe des Bandes Niederfrequente psychoanalytische Psychotherapie (mit M.G. Schmidt 2008) sowie zur intersubjektiven Betrachtung der psychoanalytischen Interaktion: Transparenz und Teilhabe, Veränderungen in der psychoanalytischen und psychodynamischen Behandlungstechnik (2017). 